surgeons, because it covers an area of great clinical importance and much recent development, which has never previously been summarised in such a concise, well-documented book. Dogma has bedevilled the use of prophylactic antibiotics even more than most aspects of surgery. The fervour with which current fashions are held by many is equalled only by the rapidity with which they are replaced. Opinions that would have produced instant failure in qualifying examinations ten years ago are considered wise, up-to-date treatment today. The clear, concise presentation of the results of objective trials, which is one of the best features of this book, is therefore most welcome. Unfortunately it becomes only too apparent on reading the evidence that it is still insufficient to be certain what is the best practice in particular circumstances. Although the theme of the book is the development of rational policy for chemoprophylaxis in surgery, in most areas we still lack the basic experimental information to achieve this aim.

This book is the result of the practical collaboration between a microbiologist and a surgeon. After the first 60 pages of general introduction, individual chapters are devoted to specific problems relating to all the surgical specialties. The concise and clear text is supplemented by particularly excellent tables of the collected results of investigations from which it is possible to draw one's own conclusions.

The nature of the basic problem in the prevention of operative infection was clearly defined by Pasteur's: "all things are hidden, obscure and debatable if the cause of the phenomena be unknown, but everything is clear if this cause be known". Thanks to Pasteur and Lister, we now know that infection is usually caused by bacteria, but we are still largely ignorant why one patient develops a postoperative infection whilst another does not. As usual in such circumstances, we take recourse to postulating a multifactorial aetiology. The resulting confusion is not helped by statements such as this one, often repeated in this book, that the incidence of infection is greatly increased if a drain is used. Surely it is just as likely that a drain is inserted when, for various reasons, the surgeon fears infection.

Perhaps the most important message that comes out of this book is that we should all monitor and carefully audit our peri-operative infection rate. The data presented will encourage us to question many of the myths relating to peri-operative infection, for instance the increased risk of infection in malnourished patients or in well controlled diabetics, neither of which has been supported by objective evidence. It is perhaps unfortunate that the authors are occasionally themselves slightly uncritically overenthusiastic. For instance, it was only 15 years ago that Brannett and Cole first advocated a short peri-operative course of antibiotics and it is only in the last year that a single intravenous dose has been described as possibly equally adequate; nevertheless the authors suggest that this is now definitely the recommended treatment. Finally, my only very minor quibble with this otherwise excellent book is that it seems slightly ungracious to write a book on infection in surgery, with several hundreds of references, without once mentioning Semmelweiss.

J. A. Dormandy

\title{
Candida and candidosis
}

By F. C. Odds. 1979. Leicester: Leicester University Press. Pp.xvii and 382. $£ 16.00$.

Since the publication of the classical monograph on Candida albicans by H. I. Winner and Rosalinde Hurley in 1964, the literature on Candida and candidosis has become so voluminous that this review by F. C. Odds must be welcomed as timely and apposite. Medical microbiologists and libraries possessing a copy of "Winner and Hurley" are well advised to purchase "Candida and candidosis" as a valuable up-to-date supplement.

In his preface Dr Odds states that "some 200 to 300 papers on candidosis appear annually in the world scientific and medical literature". The volume lists 2265 references and because many were published before "Winner and Hurley" these are significantly fewer than might be expected. Clearly, Dr Odds has adopted a policy of omission for some of those articles on Candida which once published are seldom consulted again.

There are chapters on biological aspects of yeast pathogens, on structure, physiology and biochemistry of Candida, on morphogenesis, laboratory identification, ecology and epidemi- 
ology, and on factors that predispose the host to candidosis. There are descriptions of candidosis of the oropharynx, the genitalia, the skin, nails and external sites, the gastrointestinal tract, the respiratory tract and the urinary tract, as well as of candidosis in veterinary medicine. There are also chapters on acute disseminated candidosis, pathogenesis of candidosis, antifungal agents, and, what the author terms, serodiagnosis. There is a useful data chart for the identification of yeasts in the clinical laboratory.

While I myself prefer "serology in the diagnosis of disease" to "serodiagnosis", and find the use of exclamation marks, as in the preface, jarring, nevertheless, the author presents, in a clear concise manner, a wealth of information in just 251 pages of text.

The colour plates are excellent, including the coloured scanning electromicrograph on the dust jacket, but the black and white illustrations in the text are poorly produced; a dust jacket is soon torn or mislaid, and is often discarded when the book is placed in a library, and the excellent scanning picture would have been better used as a frontispiece. A colourful dust jacket may enhance the sales of a romantic novel, but the scientific and medical interest of this book is such that it will sell itself. It is not expensive and is highly commended as a valuable reference source.

R. R. Davies

\section{Immunocytochemistry}

By L. A. Sternberger. 1979. 2nd edition. New York, Chichester, Brisbane, Toronto: John Wiley \& Sons. Pp. xii and 354. $£ 19.50$.

This book deals with the use of labelled antibody for the detection of antigen and has been compiled as a laboratory handbook for the specialist cytochemist. The author has given an excellent account of the preparation and application of fluorochrome-, enzyme- and ferritinlabelled antibodies with enough practical detail to make it only rarely necessary to consult the original sources quoted at the end of each chapter.

Surprisingly, although autoradiography is introduced conceptually, its use is not discussed in any way that does justice to this powerful technique. Indeed, it would have been better for the author to include a chapter on autoradiography rather than to discuss areas of fundamental immunology that neither offer anything to cytochemistry nor have depended upon cytochemical techniques for their development.

For the reviewer, the true value of this book lies in the last four of its ten chapters. These deal with the application of labelled-antibody techniques to neurochemistry, endocrinology, surgical cytochemistry, retrospective pathology and the study of autoimmune diseases. The book is well illustrated, contains a workable index and is likely to be as well received as its first edition.

L. HUDSON 